\title{
Commentary: Three-dimensional printing: Reshaping opportunities in congenital cardiac surgery
}

\author{
Bahaaldin Alsoufi, MD
}

\author{
From the Department of Cardiovascular and Thoracic Surgery, University of Louisville School of Medicine, Nor- \\ ton Children's Hospital, Louisville, Ky. \\ Disclosures: Author has nothing to disclose with regard to commercial support. \\ Received for publication Dec 26, 2018; accepted for publication Dec 26, 2018; available ahead of print Jan 31, \\ 2019. \\ Address for reprints: Bahaaldin Alsouf, MD, Department of Cardiovascular and Thoracic Surgery, University of \\ Louisville School of Medicine, Norton Children's Hospital, 201 Abraham Flexner Way, Suite 1200, Louisville, \\ KY 40202 (E-mail: balsoufi@hotmail.com). \\ J Thorac Cardiovasc Surg 2019;157:e291-2 \\ $0022-5223 / \$ 36.00$ \\ Copyright (c) 2019 by The American Association for Thoracic Surgery \\ https://doi.org/10.1016/j.jtcvs.2018.12.088
}

In the current issue of the Journal, Wang and colleagues ${ }^{1}$ from China report their successful management of severe bronchomalacia of the left main bronchus in a 9-year-old girl who presented with wheezing and recurrent pulmonary infections. After failure of endoluminal stenting approaches, the patient underwent left posterolateral thoracotomy and placement of a custom-made 3-dimensional (3D) printed (3DP) polycaprolactone scaffold to suspend the outside of that bronchus on the inner surface of the scaffold. This scaffold allowed bronchus expansion and easing of the pressure from the surrounding vascular structures (thoracic aorta and left pulmonary artery). On followup imaging, there was evidence of disintegration of this bioabsorbable scaffold, yet there was continuous expansion of the left main bronchus. This is not the first time that the value of this method has been reported, as this technique has been previously described in a pioneering work from the University of Michigan in 2013. ${ }^{2,3}$ Nonetheless, this case report asserts that advanced technology and innovative approaches have again helped overcome another challenging congenital anomaly that has stubbornly mystified surgeons for many decades. I am confident that every pediatric cardiac surgeon has been humbled in his or her career while attempting to treat patients with bronchomalacia or bronchial compression after surgical repair of conotruncal anomalies, aortic arch obstruction, vascular rings, and so on. I have always worried about airway obstruction complicating aortic arch surgery (eg, Norwood operation, interrupted aortic arch repair), and I have witnessed during my training and subsequently my practice a handful of patients who died as a result of this dreaded complication despite an otherwise successful anatomic repair. Traditional approaches, such as tracheostomy and chronic mechanical ventilation, endoluminal stenting, or surgical relief of airway compression (aortopexy, arterial

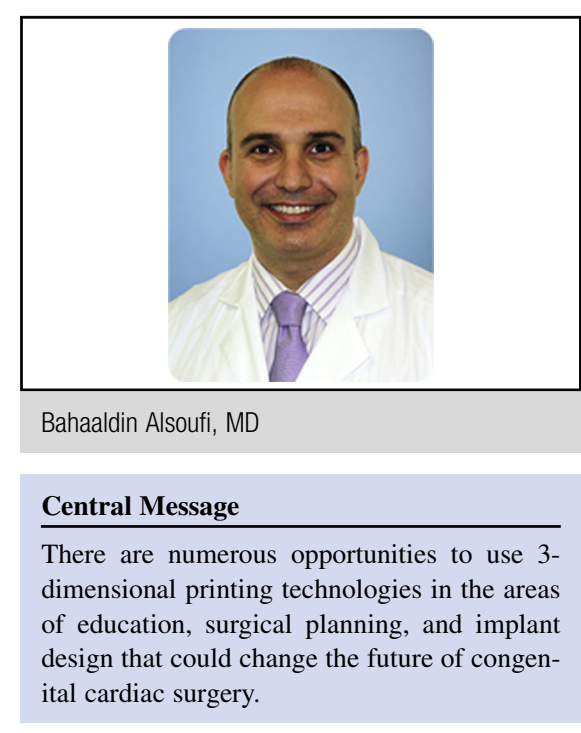

See Article page e287.

suspension, tracheoplasty, etc), have all been associated with significant complications and high failure rates. This new tool in our armamentarium is exciting, and it seems to be a both effective and reproducible way to manage a traditionally perplexing problem.

This innovative strategy that allowed creation of an individualized, biodegradable scaffold tailored to the patient's specific anatomy is attributable both to inventive surgical approaches and, more importantly, to technologic advances. These high-tech developments include superior imaging modalities with $3 \mathrm{D}$ reconstruction capabilities, progressive 3DP technology, and the ability to use biodegradable material, thus permitting future airway growth. Among those, $3 \mathrm{DP}$ seems to be the most fascinating and the most promising. Although 3DP has been in use since the 1980s, mainly in the aerospace and automotive industries, this use was subsequently followed by several applications in health care, mainly for medical implants. When coupled with modern printers, advanced imaging capabilities, and the concept of a biodegradable material as a substrate, the potential role of 3DP in the management of congenital heart disease rapidly expanded.

One common use of 3DP in congenital heart disease is the creation of a physical model that is based on a $3 \mathrm{D}$ reconstruction of patient's anatomy, allowing better understanding of complex defects, spatial relationships, and actual dimensions 
of cardiac and extracardiac structures. These models, although currently restricted by the inability to account for chordal structures, can be used as part of the decisionmaking algorithm in the preoperative planning for potential intracardiac repair of complex defects. A recent prospective, multicenter study demonstrated that 3DP models led to change in the surgical strategy in 19 of 40 cases. ${ }^{4,5}$ Moreover, combining 3DP technology with the use of virtual surgery can potentially allow physical examination of the spatial relationship of an intracardiac baffle or device in relation to the valves, ventricles, and surrounding structures. In addition to their clinical applications, these models unquestionably have a great educational value to explain the morphology of complicated congenital defects, to facilitate communication with the families, and, finally, to teach congenital cardiac surgery to trainees.

The second important application of 3DP is the creation of custom-made, patient-specific cardiac and extracardiac implants, similar to the referenced case report by Wang and colleagues. ${ }^{1}$ Those implants have been used in adult maxillofacial, orthopedic and neurosurgery. In those situations, static rather than biodegradable material was used, because growth was not required, and long-term stability was clinically indicated. The use of biodegradable material further increases 3DP utility to provide implants that would eventually dissolve after serving their purpose and would therefore allow for growth. ${ }^{2,3,6,7}$ Considerable preparation is naturally required to establish patient's anatomy, to calculate the desired size and shape of the implant, and to choose the material that would allow stability of the implant for the intended duration plus timely dissolution to permit future growth. The pioneering work from Michigan, ${ }^{2,3}$ echoed by this case report from China, ${ }^{1}$ is an excellent example of the successful collaboration of physicians, scientists, and engineers to tackle a challenging problem.

The science of 3DP is in its infancy; however, the opportunities it presents are enormous in congenital heart surgery. It is likely that 3DP will become the ultimate tool to treat difficult pathologies, such as bronchomalacia, or to create necessary implants. Improved imaging modalities that would enable accurate imaging of valvular and chordal structures might further increase the utility of 3DP to facilitate design and manufacture of pediatric valves. There will be numerous regulatory and methodologic hurdles to overcome; however, as shown in the case of bronchomalacia, persistence is a guarantee that results are inevitable, and I am confident that 3DP will transform the way that we treat many congenital cardiac disorders in the near future.

\section{References}

1. Wang L, Liu W, He J, Li X, Huang L. Treatment of bronchomalacia using threedimensional printed polycaprolactone scaffold in a pediatric patient. J Thorac Cardiovasc Surg. 2019;157:e287-90

2. Zopf DA, Hollister SJ, Nelson ME, Ohye RG, Green GE. Bioresorbable airway splint created with a three-dimensional printer. N Engl J Med. 2013; 368:2043-5.

3. Morrison RJ, Sengupta S, Flanangan CL, Ohye RG, Hollister SJ, Green GE. Treatment of severe acquired tracheomalacia with a patient-specific, 3D-printed, permanent tracheal splint. JAMA Otolaryngol Head Neck Surg. 2017;143:523-5.

4. Valverde I, Gomez-Ciriza G, Hussain T, Suarez-Mejias C, Velasco-Forte MN, Byrne N, et al. Three-dimensional printed models for surgical planning of complex congenital heart defects: an international multicentre study. Eur J Cardiothorac Surg. 2017:52:1139-48.

5. Milano EG, Capelli C, Wray J, Biffi B, Layton S, Lee M, et al. Current and future applications of 3D printing in congenital cardiology and cardiac surgery. Br J Radiol. November 1, 2018 [Epub ahead of print].

6. Farooqi KM, Cooper C, Chelliah A, Saeed O, Chai PJ, Jambawalikar SR, et al. 3D printing and heart failure: the present and the future. JACC Heart Fail. December 10, 2018 [E-pub ahead of print].

7. Morrison RJ, Kashlan KN, Flanangan CL, Wright JK, Green GE, Hollister SJ, et al. Regulatory considerations in the design and manufacturing of implantable 3D-printed medical devices. Clin Transl Sci. 2015;8:594-600. 